\title{
THE DISTRIBUTION OF I'131 LABELED HUMAN SERUM ALBUMIN INTRODUCED INTO ASCITIC FLUID: ANALYSIS OF THE KINETICS OF A THREE COMPARTMENT CATENARY TRANSFER SYSTEM IN MAN AND SPECULATIONS ON POSSIBLE SITES OF DEGRADATION
}

\author{
BY SOLOMON A. BERSON AND ROSALYN S. YALOW
}

(From the Radioisotope Unit of the Veterans Administration Hospital, Bronx, N. Y.)

(Submitted for publication July 20, 1953; accepted November 25, 1953)

The use of ${ }^{181}$ labeled human serum albumin as a tracer has permitted the study of transfer of serum albumin between plasma and extravascular compartments. Distribution of intravenously administered iodoalbumin between plasma and ascitic fluid has been previously analyzed in terms of a simplified two compartment system $(1,2)$. In the present investigation iodoalbumin was administered intraperitoneally and the kinetics of distribution were analyzed by reference to a three compartment schematic model composed of ascitic fluid, plasma and extravascular spaces other than peritoneal cavity. The time course of excretion of the radioactive iodine released by degradation of the labeled albumin was also followed in an attempt to localize the site of metabolic breakdown of iodoalbumin.

\section{METHODS}

The subject of the distribution study was a 50 -year-old white man, who, over the preceding eight months, had been subjected to repeated paracenteses for ascites secondary to Laennec's cirrhosis. Ten drops of Lugol's solution was administered three times a day throughout the period of observations to inhibit uptake by the thyroid of iodine released from the degraded iodoalbumin. Following administration of $112 \mu \mathrm{c}$ of dialyzed $I^{181}$-tagged human serum albumin into the peritoneal cavity, ascitic fluid and heparinized plasma samples were obtained at almost daily intervals and complete urine collections were made. Under these conditions, loss of radioiodine from the body is almost entirely accounted for through renal excretion (2). Furthermore, owing to the relative rapidity of excretion as compared to degradation, the curve of urinary excretion of radioiodide may be considered as virtually identical with the curve of iodoalbumin degradation (2).

Radioactivity in plasma, ascitic fluid, and urine was assayed as previously described (2). Albumin concentrations in plasma and ascitic fluid were determined both by the micro-Kjeldahl method employing the technique of Kingsley (3) for the precipitation of proteins and by electrophoretic analysis. Previous observations (2), that at least 98 per cent of radioactivity in ascitic fluid and plasma is precipitable with cold 10 per cent trichloracetic acid, were confirmed. The patient did not have detectable proteinuria and radioactivity in the urine was not precipitated by trichloracetic acid even after carrier albumin was added.

The ascitic fluid volume was calculated from the 15minute space of distribution of the $I^{131}$ labeled albumin and was determined at the beginning and at the end of the experiment. Shaking and massage of the abdomen facilitated mixing. Plasma volume had been measured previously (2) with $I^{131}$ labeled albumin (4) and was assumed to be unchanged.

\section{SCHEMATIC MODEL}

Following intraperitoneal injection, the fluid compartments are represented as components of a catenary system ${ }^{1}$ in which the peritoneal cavity and extravascular space (other than peritoneal cavity) are end compartments communicating with the intervening plasma compartment, as shown in Figure 1. Although it is presumed that albumin leaves the blood stream by passage through the capillary walls, it is probable that return is chiefly by way of the lymphatics under normal conditions. The boundary for return from ascitic fluid to plasma would then involve an intervening shunt, the lymphatic vessels, which is considered a part of the extravascular space. In addition, another shunt space is the right pleural cavity which may receive albumin from the peritoneal cavity in a oneway transfer. However, the quantity of albumin within these shunts at any time is small compared to that in any of the three major compartments and is therefore neglected.

Following intravenous administration of $I^{181}$ albumin to subjects without abnormal fluid accumulations, such as ascites, a 2 component curve of disappearance from plasma was previously observed in most instances (2). This curve possibly represents a mammillary system in which the central reservoir, plasma, is in communication with

1 The term catenary is used by Sheppard \& Householder (5) to describe a series of compartments communicating endwise with each other. The term mammillary is used to describe a number of peripheral compartments in communication with a central reservoir.

2 The extravascular space of serum albumin distribution is thought to be composed chiefly of fluid in the interstitial (extracellular) space and lymphatic channels but the possibility of some intracellular localizations is not excluded. 


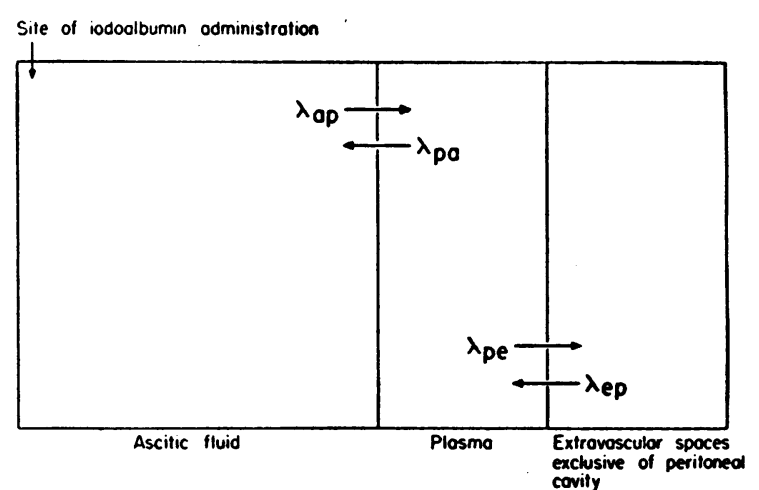

Fig. 1. Schematic Model for Transfer of Im ALBUMIN ADMINISTERED INTRAPERITONEALLY

two groups of extravascular spaces with different average net transfer rates. In the present study it was felt that consideration of more than a single rate of transfer between plasma and extravascular space would introduce unresolvable complications into the analysis. Therefore, the extravascular spaces were assumed to comprise a single compartment communicating with plasma by a single transfer rate representing an average of all such transfer rates.

Let $A, P$, and $E$ be the quantities of exchangeable endogenous albumin in ascitic fluid, plasma and extravascular space, respectively, $A^{*}$ be the total amount of $I^{131}$ labeled albumin introduced into the ascitic fluid compartment and $A^{*}, P^{*}$, and $E^{*}$ be the fractions of $A^{*}$ in the respective compartments at any time, $t$. Let $\lambda_{\mathrm{ap}}, \lambda_{\mathrm{pa}}, \lambda_{\mathrm{pe}}$, and $\lambda_{\text {ep }}$ be the rate constants of transfer between the compartments in the directions indicated by the order of the subscript designations, and $\mathrm{A}^{*}, \mathrm{P}_{\infty}^{*}$, and $\mathrm{E}_{\infty}^{*_{\infty}}$ be the fractions of $\mathrm{A}^{*_{0}}$ in the respective compartments at distribution equilibrium..$^{3}$

In the "steady state," the quantities of endogenous albumin in the various compartments remain unchanged. Assuming that the amounts being transferred in each direction remain constant and that the labeled and unlabeled molecules behave identically, then

$$
\begin{aligned}
& \frac{d A^{*}}{d t}=-\lambda_{a p} A^{*}+\lambda_{p a} P^{*} \\
& \frac{d P^{*}}{d t}=-\lambda_{p a} P^{*}+\lambda_{a p} A^{*}-\lambda_{p 0} P^{*}+\lambda_{p p} E^{*} \\
& \frac{d E^{*}}{d t}=-\lambda_{p p} E^{*}+\lambda_{p 0} P^{*}
\end{aligned}
$$

Employing the conditions that

$$
A^{*}=A^{*}, P^{*}=0, E^{*}=0 \quad \text { when } t=0
$$

and

$$
A^{*}=A_{\infty}^{*}, P^{*}=P_{\infty}^{*}, E^{*}=E_{\infty}^{*} \quad \text { when } t=\infty,
$$

In this paper distribution equilibrium is defined as a dynamic state in which transfer of the labeled albumin from one compartment to another per unit time is balanced by transfer of an equal amount in the reverse direction. the system of simultaneous linear differential equations, (1) (2) (3), is solved for $A^{*}, P^{*}$ and $E^{*}$ by standard methods (Appendix A).

$$
\begin{aligned}
& A^{*}=A^{*}+c_{1} e^{-\lambda_{1} t}+c_{2} e^{-\lambda_{2} t} \\
& P^{*}=P_{\infty}^{*}-\left(k_{1}+c_{1}\right) e^{-\lambda_{1} t}-\left(k_{2}+c_{2}\right) e^{-\lambda_{2} t} \\
& E^{*}=E^{*}+k_{1} e^{-\lambda_{1} t}+k_{2} e^{-\lambda_{2} t}
\end{aligned}
$$

where

$$
\begin{aligned}
& \lambda_{1,} \lambda_{2}=\frac{1}{2}\left\{\left(\lambda_{p a}+\lambda_{p e}+\lambda_{2 p}+\lambda_{e p}\right) \pm\left[\left(\lambda_{p a}+\lambda_{p e}\right.\right.\right. \\
& \left.\left.\left.+\lambda_{\text {ap }}+\lambda_{\text {op }}\right)^{2}-4\left(\lambda_{\mathrm{sp}} \lambda_{\mathrm{pe}}+\lambda_{\mathrm{ep}} \lambda_{\mathrm{ps}}+\lambda_{\mathrm{sp}} \lambda_{\mathrm{op}}\right)\right]\right\} \\
& c_{1}=\frac{\lambda_{2}\left(A_{0}^{*}-A_{\infty}^{*}\right)-\lambda_{a p} A_{0}^{*}}{\lambda_{2}-\lambda_{1}} \quad k_{1}=-\frac{\lambda_{2} E^{*}}{\lambda_{2}-\lambda_{1}} \\
& c_{2}=-\frac{\lambda_{1}\left(A *_{0}-A_{\infty}^{*}\right)-\lambda_{a p} A_{0}^{*}}{\lambda_{2}-\lambda_{1}} \quad k_{2}=\frac{\lambda_{1} E^{*}}{\lambda_{2}-\lambda_{1}}
\end{aligned}
$$

\section{ANALYSIS OF OBSERVED DATA}

The amounts of $I^{131}$-albumin in the ascitic fluid and plasma compartments at any time, $t$, were calculated as the products of the compartment volumes and the respective concentrations of $I^{131}$-albumin. The previously determined plasma volume was assumed to remain unchanged throughout the period of observations. However, over this 22-day period, the ascitic fluid volume increased from 14.5 to 21 liters. This increase, averaging 295 milliliters per day, or between $1 \frac{1}{2}$ per cent and 2 per cent per day, was assumed to proceed linearly with time, since the subject's weight increased gradually over this period. This necessary approximation probably did not introduce an error of more than 5 per

TABLE I

Ascitic fluid and plasma concentrations of serum albumin*

\begin{tabular}{ccc}
\hline \hline $\begin{array}{c}\text { Day of } \\
\text { study }\end{array}$ & $\begin{array}{c}\text { Ascitic } \\
\text { fluid } \\
\text { Gm./100 } \mathrm{ml} .\end{array}$ & $\begin{array}{c}\text { Plasma } \\
\text { Gm./100 ml. }\end{array}$ \\
\hline 5 & 1.50 & 2.95 \\
6 & 1.50 & 2.85 \\
7 & 1.51 & \\
8 & 1.58 & 3.04 \\
12 & 1.49 & 3.00 \\
13 & 1.44 & 2.98 \\
14 & 1.48 & 2.98 \\
15 & 1.45 & 2.85 \\
16 & 1.51 & 3.05 \\
19 & 1.47 & \\
20 & & \\
\hline
\end{tabular}

* These values were obtained from Kjeldahl determinations. Electrophoresis gave absolute values which were somewhat lower but with the same ratio between plasma and ascitic fluid. 
cent into the calculation of total ascitic fluid $\mathrm{I}^{131}$ albumin at any time.

Then,

$\mathrm{P}^{*}=$ plasma volume $(\mathrm{ml}$.$) \times fraction of \mathrm{A}_{0} /$ ml. plasma

$\mathrm{A}^{*}=$ ascitic fluid volume $(\mathrm{ml}$.$) \times fraction of$ $A *_{0} / \mathrm{ml}$. ascitic fluid

ascitic fluid volume $(\mathrm{ml})=14,.500+295 \mathrm{t}$ where $t$ is the number of days from the beginning of the study.

The total quantity of extravascular iodoalbumin was calculated as the total iodoalbumin remaining within the body, less the sum of ascitic fluid and plasma iodoalbumin. The amount retained within the body $\left(A^{*}{ }_{\text {ret }}\right)$ at any time, $t$, was taken as the dose administered less the cumulative renal excretion up to that time.
Then,

$$
\begin{aligned}
& A^{*}{ }_{0 \text { ret }}=A^{*}-U^{*} \\
& E^{*}=A_{0 \text { ret }}^{*}-\left(P^{*}+A^{*}\right)
\end{aligned}
$$

where $\mathrm{U}^{*}$ is the cumulative urine excretion.

Direct determinations of extravascular $\mathrm{I}^{\mathbf{1 3 1}}$ albumin concentration were not feasible. However, such determinations would not have permitted estimates of total extravascular I $^{131}$-albumin since there are wide variations in albumin concentration in different portions of this compartment and the volume of each portion is not known. The concentrations of albumin in ascitic fluid and plasma remained essentially constant (Table I). When the relative specific activities of the ascitic fluid, plasma and extravascular space were plotted as a function of time on semi-

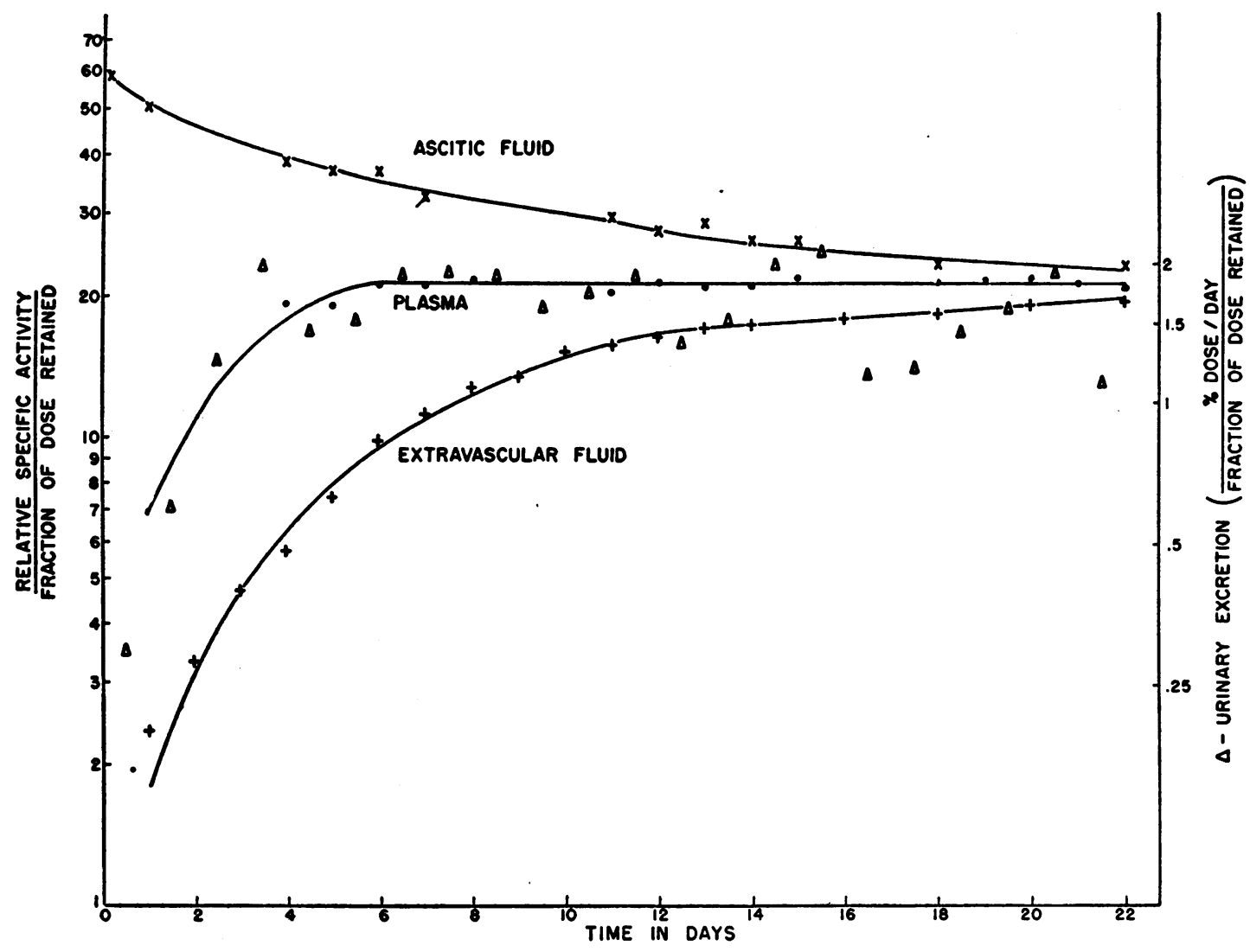

Fig. 2. Relative Specific Activities of I ${ }^{131}$ Albumin in Ascitic Fluid, Plasma and Extravascular Fluid and URinary Excretion of Radioactivity as a Function of Time Following Intraperitoneal InJECTION OF I'131 AlBumin

Points on the ascitic fluid (A) and plasma (P) curves are derived from experimentally determined values. Points on the extravascular fluid curve were calculated as described in Figure 3 and the text. Total endogenous albumin in plasma was 137 grams, in extravascular fluid 157 grams, and in ascitic fluid 217 grams at onset increasing to 316 grams at conclusion of study. 
logarithmic paper (Figure 2), it was obvious that equilibrium conditions were still not satisfied even at the end of 22 days since the specific activity in the ascitic fluid was still greater than that of the plasma at that time. However, the long plateau maintained by the plasma specific activity indicates that the plasma had virtually reached its equilibrium specific activity at that time.

Since the specific activity of the plasma at equilibrium defines the specific activities at equilibrium for the other two compartments as well, the predicted radioactivities at equilibrium for the ascitic fluid and extravascular space may be obtained at any time from the values for total albumin in each of these compartments. Since the quantity of ascitic fluid albumin increased throughout the period, however, the equilibrium values did not remain constant as in an ideal closed steady state system and some consideration had to be given to the source of this increased ascitic fluid albumin. The patient had been relieved of approximately 1000 grams of albumin by repeated paracenteses over a five-month period. When this is compared with his total exchangeable albumin (excluding ascitic fluid albumin) of about 300 grams, it is clear that, much, if not all, of the observed increase in ascitic fluid albumin was due to newly formed albumin in excess of albumin degraded. That little of the increase could have been due to shifting from plasma and extravascular space is substantiated by the maintenance of a constant plasma concentration during this period (Table I) and a relatively fixed quantity of albumin outside the peritoneal cavity. This is indicated by the following calculations. On the last day of observations total exchangeable albumin was 609 grams (vide infra) and ascitic fluid albumin was 315 grams. The difference, 294 grams represents the sum of plasma albumin (137 grams) and albumin in the extravascular space (157 grams). These figures are to be compared with the previously determined value of 385 grams total exchangeable albumin at a time when the ascitic fluid contained 75 grams (2). Thus, this subject maintained about 300 grams in plasma and extravascular space other than ascitic fluid.

For the foregoing reasons the total albumin contents of the plasma and extravascular compartment were considered to remain unchanged, the accumulation of ascitic fluid albumin being associated with an increase in the total exchangeable albumin. The total exchangeable albumin on the last day of observations was calculated as the retained dose divided by the specific activity of plasma albumin. This amounted to 609 grams. Total exchangeable albumin (TEA) on each preceding day was then estimated by subtracting from this value the daily increments of ascitic fluid albumin. Equilibrium values for each compartment at every point were then calculated from the following formulas.

$$
\begin{aligned}
A^{*} \text { oq }_{t} & =\frac{A_{t}}{(T E A)_{t}} \\
E^{*}{ }_{o q_{t}} & =\frac{E_{t}}{(T E A)_{t}} \\
P^{*}{ }_{\text {oq }} & =\frac{P_{t}}{(T E A)_{t}}
\end{aligned}
$$

$A^{*}{ } q_{t}, E^{*}{ } q_{t}$ and $P^{*}{ } q_{t}$, therefore, represent the fractions of $A^{*} 0$ ret which would be present in the respective compartments at time $t$ if the tracer were instantaneously distributed throughout the system at that time.

The fractions of $A * 0$ ret in each of the compartments and the calculated values for $A^{*}{ }^{*} q_{6}, P^{*}{ }^{*} q_{t}$ and $E^{*}{ }^{*}$, are shown in Figure 3. The curves were resolved into their various exponential components by subtracting the values for each point from the respective equilibrium values and plotting the differences. Following extrapolation of the straight line component of each of these curves to zero time, the same process was repeated for the evaluation of the second exponential. The coefficients were determined from the vertical axis intercepts and the values for $\lambda_{1}$ and $\lambda_{2}$ were calculated from the means of the decay constants of each of the exponentials in the three curves. There was reasonable agreement of values for each of the decay constants determined from the three curves.

The observation that $c_{1}+c_{2}+A_{e q_{t}}$ is about 5 per cent lower than $A_{0}$, may be attributable to mixing of the originally injected material with only 95 per cent of the entire volume of ascitic fluid during the first fifteen minutes. 


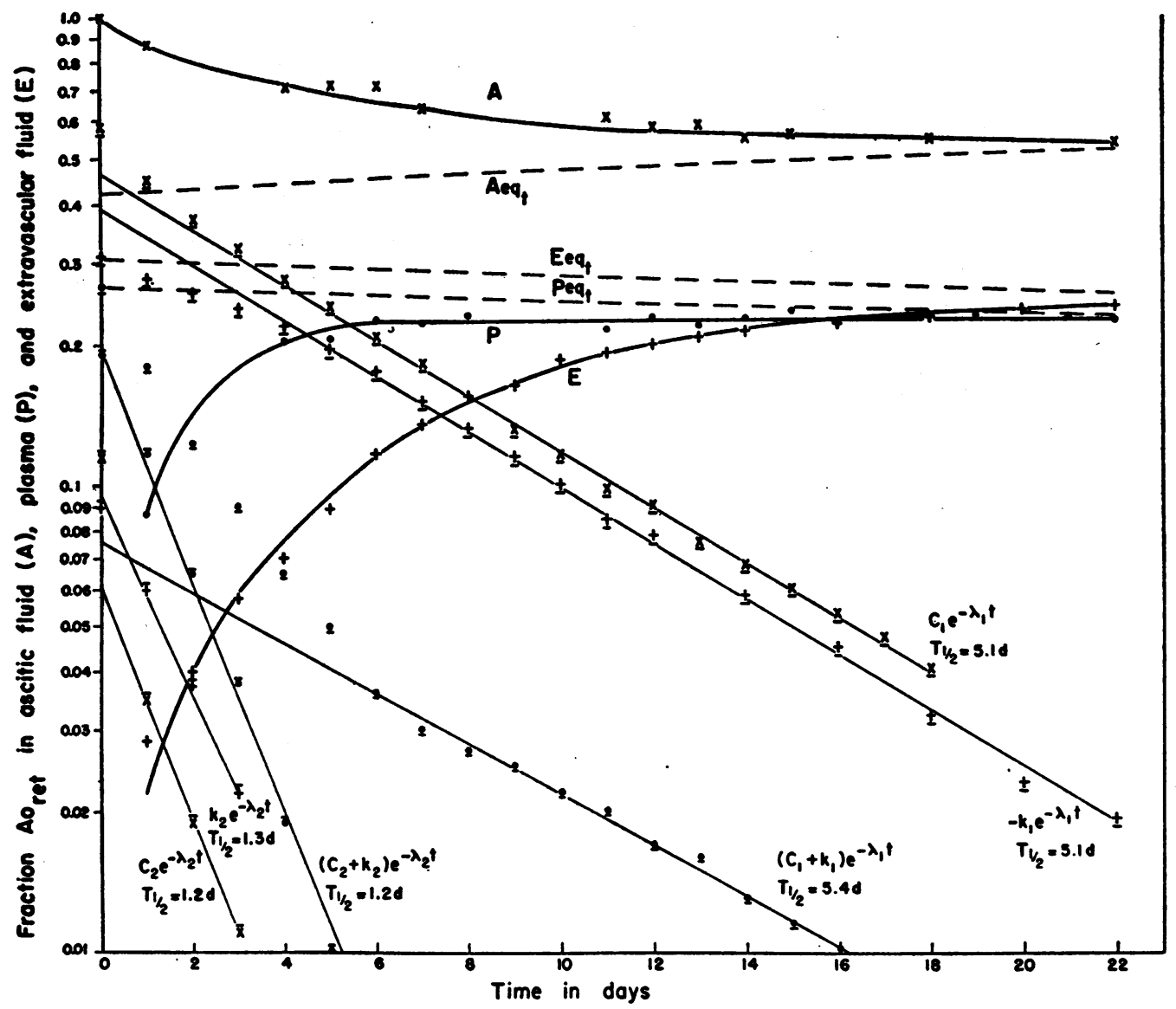

Fig. 3. Graphic Analysis of the Distribution Curves

Fraction of dose retained in ascitic fluid (A), plasma (P) and extravascular space (E) has been plotted as a function of time following intraperitoneal administration of $I^{131}$ albumin. Points on curves $A$ and $P$ are derived from experimentally determined values. Points on curve $E$ are calculated from the relationship $A_{o_{\text {ret }}}=A_{t}+P_{t}+E_{t}$. Calculations for $A_{\text {eq }}, P_{\text {eqt }}$ and $E_{\text {oq }}$ are described in the text. Points underscored with a bar are obtained by subtracting from the respective equilibrium values, simultaneous points on curves $A, P$, and $E$. Points on the extrapolated terminal straight line component of the new curves thus produced were then subtracted from these curves to give the points overscored with a bar.

Quantitative evaluation of albumin transfer between compartments and consideration of the errors involved

The rate constants $\lambda_{\mathrm{ap}}, \lambda_{\mathrm{pa}}, \lambda_{\mathrm{op}}$ and $\lambda_{\mathrm{pe}}$ can be mathematically expressed (Appendix B) in terms of the coefficients and exponents of equations 4 to 6 which are obtained from analysis of the experimentally determined curves in Figure 3. However, the values for the rate constants, calculated in this manner, are quantitatively valid only within the steady state conditions imposed by the theoretical analysis. The approximations necessitated by the departure from these conditions induces certain discre- pancies into the derived quantitative data which may be considered as follows.

Since the quantity of endogenous albumin in the ascitic fluid increased, the steady state conditions were not fully realized. However, it is reasonable to assume, as in the theoretical analysis, that during the period of observations the absolute quantities of albumin crossing the boundaries in each direction per unit time did not change significantly; that is, that the products of the rate constants and the total quantities of albumin in the respective compartments remained constant. From the evidence pointing to a fairly constant amount of albumin in 
plasma and extravascular space, the relative constancy of $\lambda_{\mathrm{pa}}, \lambda_{\mathrm{pe}}$, and $\lambda_{\mathrm{ep}}$ may be accepted. But $\lambda_{\mathrm{ap}}$ must have decreased proportionately as the ascitic fluid albumin increased. The constancy of $\lambda_{1}$ and $\lambda_{2}$, as implied in Figure 3 , is, therefore, only approximate. This lack of constancy is difficult to detect, however, since a 45 per cent decrease in $\lambda_{\text {ap }}$ produces only a 14 per cent change in $\lambda_{1}$ and less than a 1 per cent change in $\lambda_{2}$.

The steady state condition that $\lambda_{\mathrm{pa}} \mathrm{P}=\lambda_{\mathrm{ap}} \mathrm{A}$ is also not satisfied when there is a net increase in ascitic fluid albumin. Since this increase averaged about 4.4 grams daily, the experimental condition for the albumin transferred per day is $\lambda_{\mathrm{pa}} \mathrm{P}=\lambda_{\mathrm{ap}} \mathrm{A}+4.4$. The value obtained for the rate of albumin transfer from plasma to ascitic fluid is then approximately 24 grams per day (Appendix B) and that for transfer in the reverse direction about 20 grams per day.

From these considerations it may be concluded that, because of the approximations required by the absence of steady state conditions (which rarely exist in disease states), the values derived even from a reasonably complete analysis of available data, can be considered only roughly quantitative. Any further simplifica- tion of the reference scheme is likely to yield even less precise quantitative results. An example of this is presented in the values obtained for albumin transfer between plasma and extravascular fluid. It is calculated that approximately 30 grams daily passed in each direction between plasma and extravascular fluid (Appendix B). However, following intravenous administration of iodoalbumin in subjects without ascites, the initial fall in plasma concentration is of the order of 5 per cent per hour $(2,4)$, which indicates a transfer into extravascular fluid of over 100 grams albumin per day. It is possible that exchange between plasma and extravascular space was abnormally low in this cirrhotic subject because of increased tissue tension resulting from edema. Yet large errors can result from the simplifying assumption of a single extravascular compartment communicating with plasma at a single transfer rate. For example, a small extravascular compartment equilibrating rapidly with plasma could well escape detection in this type of analysis and yet be responsible for a quantitatively greater albumin traffic to and from plasma than all the rest of extravascular space.

From the theoretical point of view a complete

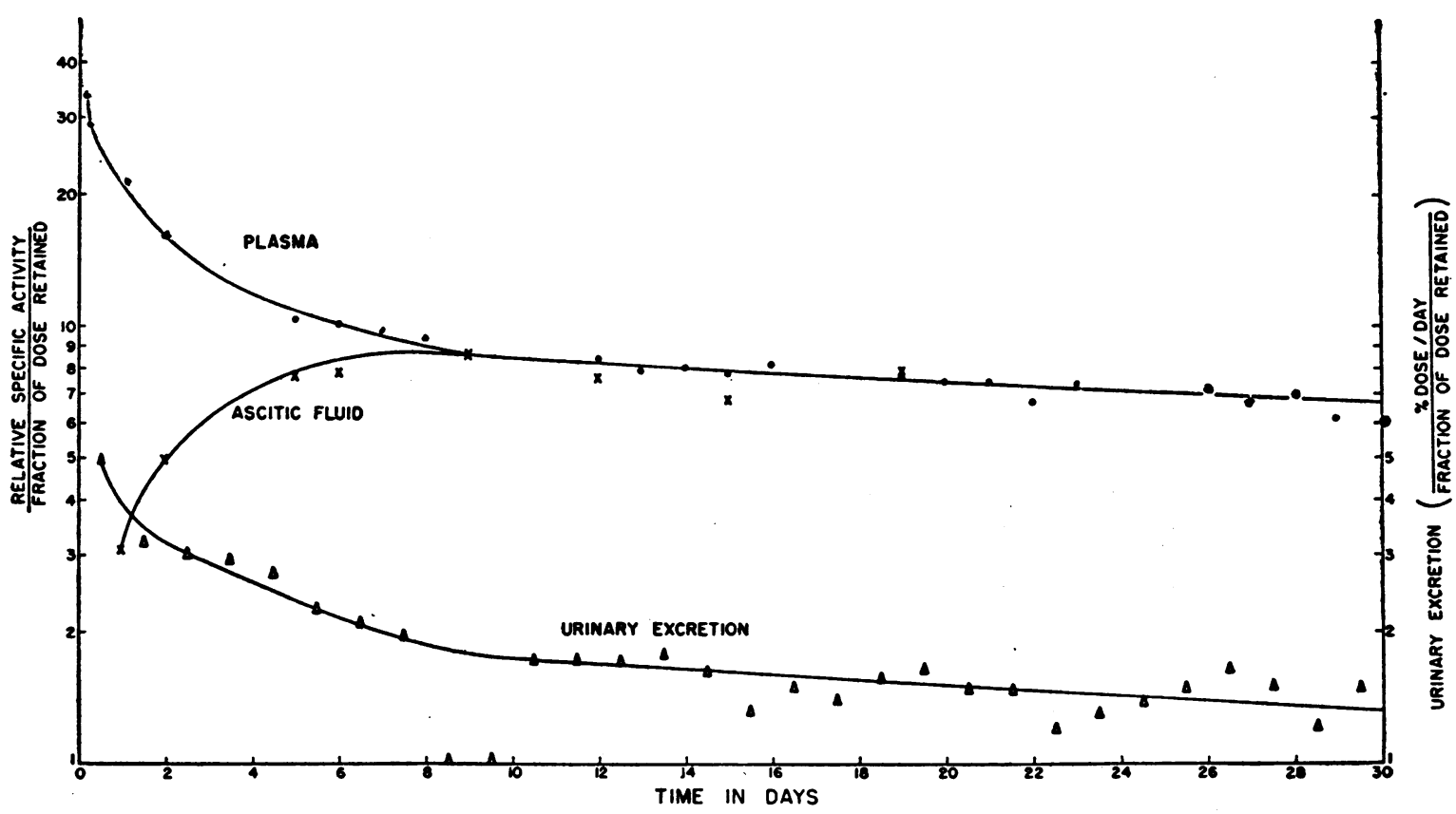

Fig. 4. Specific Activities of I ${ }^{131}$ Albumin in Ascitic Fluid and Plasma and Urinary Excretion of Radioactivity as a Function of Time Following the Intravenous Administration of I ${ }^{12}$ Albumin 
kinetic analysis of the closed steady state schematic model requires only the curve of radioactivity concentration in the ascitic fluid and a knowledge of the total amounts of albumin in the ascitic fluid and plasma. In practice, however, even small experimental errors are sufficient to lead to gross miscalculations when such limited data alone are available. The failure to obtain immediate complete mixing in the ascitic fluid may lead to an early steep fall in concentration of radioactivity which, if observations were confined to this compartment alone, would be interpreted as indicating a greater transfer rate than actually exists. On the other hand, as noted above, a slight experimental error may also obscure a rapid transfer component into a small compartment. Extending observations to the receiving compartments aids in discriminating between experimental errors and variations arising from the specific kinetic characteristics of the system.

\section{SITE OF IODOALBUMIN DEGRADATION}

It has previously been noted that the curve of renal excretion of radioactivity is virtually identical with the curve of degradation of iodoalbumin (2). If one compartment were the sole or major site of degradation, it would be expected that the renal excretion of radioactivity would be more or less directly proportional to the radioactivity present in the degrading compartment. In Figure 4 are shown the results of a previous experiment in the same subject where iodoalbumin was administered intravenously. Since ascitic fluid volumes were not measured directly, calculated values for the extravascular space are not shown. The renal excretion follows more closely the specific activity of the plasma than that of the ascitic fluid. In three other cirrhotic subjects with ascites where ascitic fluid concentrations of radioactivity were not assayed, but may be presumed to have shown generally similar increasing curves, the plasma and urine curves were similar to those shown here. These patterns indicate that degradation is not proportional to ascitic fluid radioactivity and, therefore, that the ascitic fluid is not a major site of degradation. A similar conclusion can be inferred from the present study, since renal excretion increased as ascitic fluid radioactivity de- creased. There is also an apparently closer correlation between the radioactivity in the plasma and renal excretion than between the radioactivity in extravascular space and renal excretion. This suggests that extravascular space as a whole is also not a major site of degradation. However, the conclusion that the parallelism of the plasma and urine curves indicates the plasma as the site of iodoalbumin catabolism must be avoided. An alternative interpretation is that a certain segment of the extravascular space in fairly rapid distribution equilibrium with the plasma may be the site of degradation. Previous studies (2) have shown that there is some extravascular compartment which exchanges rapidly with plasma. Owing to some lag in excretion of degraded radioactivity and the presence of small amounts of proteinfree radioactivity even after prolonged dialysis, the renal excretions over the first few hours cannot be taken so seriously as to discriminate between the plasma and the rapidly equilibrating extravascular compartments. The possibility that this extravascular compartment is located in an intracellular site cannot be excluded.

\section{DISCUSSION}

A general mathematical approach to the problem of tracer mixing in multicompartment systems has been presented by Sheppard and Householder (5). The present study illustrates the practical difficulties encountered in attempting to apply a theoretical analysis to a specific problem, that of the distribution of intraperitoneally administered $I^{181}$ tagged albumin. The failure of the biological system to remain conveniently constant introduced discrepancies of moderate degree into the derived data. The simplifying reduction of all extravascular spaces into a single compartment necessitated by the lack of information regarding the various extravascular compartments and the infeasibility of repeated sampling of them may be responsible for errors of greater magnitude. It would appear to be even less justified to neglect the extravascular compartments entirely as well as the consideration of changing volumes, as in the approach of Schoenberger, Kroll, Sakamoto, and Kark (1). These authors have concluded that alterations in permeability are responsible for 
changes in albumin transfer rates observed in cirrhotic patients under therapy (6). In view of the obstacles which oppose the accurate evaluation of these transfer rates it seems a bit hazardous to rely too heavily on the quantitative aspects of data calculated by reference to oversimplified systems.

In spite of this conservative approach to the significance of values obtained for albumin exchange among the various compartments, certain quantitative considerations are in order. In normal subjects, extravascular space contains approximately 150 to 200 grams of albumin (2). Following intravenous administration of $I^{131}$ albumin to non-edematous subjects, the half time for equilibration with the major portion of this extravascular albumin is about 24 hours (2). It thus appears that the rate of transfer of plasma albumin to most of the extravascular compartment is normally only of the order of five times the rate of transfer of plasma albumin to ascitic fluid (24 grams per day) observed in the cirrhotic subject of the present study. Krogh (7) has estimated the total surface area of capillaries throughout the body to be about 6000 square meters. Since the total surface area of the peritoneal capillaries probably does not approach that value within a factor of more than one hundred, a conservative estimate must grant an albumin transfer rate per unit capillary surface area at least 20 times greater through the average peritoneal capillary of this subject than through the normal average extraperitoneal capillary. Each square centimeter of the peritoneal membrane itself, which cannot have a total surface area of much more than 1 square meter, is therefore the site of even considerably greater transfer activity. Data for the normal subject would appear difficult to obtain without altering normal physiological conditions.

The continued accumulation of ascitic fluid albumin in cirrhotic subjects indicates either an abnormal increase in the rate of transfer from plasma to ascitic fluid or a decrease in the rate of removal from the peritoneal cavity or both. We are not aware of any evidence bearing on obstruction of the lymphatics which drain the peritoneal cavity in cirrhosis, while the high portal venous pressure present in this condition suggests a mechanism for the first alternative.
Then, fluctuations in the rate of ascitic fluid accumulation in cirrhosis may depend chiefly on changes in portal capillary pressure (as is suggested by the occasional success of shunting operations) rather than on any change in permeability of the capillaries. It would be of interest to investigate the rate of albumin transfer in nephrotic ascites where portal venous pressure is not elevated.

It has been reported that, after intraperitoneal administration of iodoalbumin, the specific activity of ascitic fluid remains higher than that of plasma (8). This relationship is more apparent than true and in the present case owes itself to a half time of over five days for the slow component of the distribution curves. A month or more may be required for essentially complete distribution of iodoalbumin administered intraperitoneally to subjects with marked ascites.

The problem of the site of degradation remains unsolved from the data at hand. It appears as if the iodoalbumin is subject to degradation in the plasma itself or in a rapidly equilibrating extravascular compartment. Attempts to study the proteolytic effect of plasma on iodoalbumin in vitro have thus far given equivocal results.

It has previously been emphasized (2) that the metabolic degradation of $\mathrm{I}^{131}$ labeled albumin has not as yet been conclusively demonstrated to be identical with that of endogenous serum albumin. Conclusions regarding iodoalbumin metabolism can therefore only tentatively be suggested as reflecting the fate of endogenous albumin.

\section{CONCLUSIONS}

1. Following intraperitoneal administration of I $^{131}$ labeled human serum albumin, the kinetics of distribution have been analyzed mathematically with reference to a three compartment schematic model composed of ascitic fluid, plasma, and extravascular fluid.

Errors of the analysis arising from non-steady state conditions and deficiencies in the schematic model are discussed.

2. It was demonstrated that essentially complete distribution of intraperitoneally administered $I^{131}$ labeled albumin may require almost a month in the presence of marked ascites. The rate of albumin transfer from plasma to ascitic fluid was calculated to be approximately 24 
grams per day. During a 22-day period of observations the ascitic fluid showed a net increase in albumin content which averaged 4.4 grams per day. Evidence is presented which indicates that this increase represented newly formed albumin rather than a depletion of exchangeable albumin stores in plasma and extravascular fluid.

3. From considerations of relative available capillary surface areas it is concluded that the peritoneal capillary of the cirrhotic subject of the present study permitted a much greater albumin transfer out of plasma than the average capillary elsewhere in the body.

4. From a comparison of the time course of renal excretion of radioactivity with the curves of $I^{131}$ labeled albumin in ascitic fluid, plasma and extravascular fluid following intraperitoneal and intravenous administration of the labeled albumin, the site of degradation of iodoalbumin has been inferred to be the circulating plasma or some extravascular compartment equilibrating rapidly with plasma.

\section{APPENDIX $\mathrm{A}^{4}$}

Solution of the differential equations $1,2,3$

$$
\begin{aligned}
& \frac{d A}{d t}=-\lambda_{a p} A+\lambda_{p a} P \\
& \frac{d P}{d t}=-\lambda_{p a} P+\lambda_{a p} A-\lambda_{p e} P+\lambda_{o p} E \\
& \frac{d E}{d t}=-\lambda_{o p} E+\lambda_{p e} P
\end{aligned}
$$

Differentiating (1) and substituting the value for $\frac{\mathrm{dP}}{\mathrm{dt}}$ given in (2) gives

$$
\begin{aligned}
\frac{d^{2} A}{d t^{2}}=-\lambda_{2 p} \frac{d A}{d t}- & \lambda_{p Q} P\left(\lambda_{p 2}+\lambda_{p o}\right) \\
& +\lambda_{2 p} \lambda_{p q} A+\lambda_{p 2} \lambda_{p p} E
\end{aligned}
$$

Substituting the value for $\lambda_{p s} \mathrm{P}$ determined from (1), differentiating and collecting terms,

$$
\begin{aligned}
\frac{\mathrm{d}^{2} \mathrm{~A}}{\mathrm{dt}^{3}}+\frac{\mathrm{d}^{2} \mathrm{~A}}{\mathrm{dt}^{2}}\left(\lambda_{\mathrm{ap}}\right. & \left.+\lambda_{\mathrm{pa}}+\lambda_{\mathrm{po}}\right) \\
& +\frac{\mathrm{dA}}{\mathrm{dt}}\left(\lambda_{\mathrm{ap}} \lambda_{\mathrm{po}}\right)=\lambda_{\mathrm{pa}} \lambda_{\mathrm{sp}} \frac{\mathrm{dE}}{\mathrm{dt}}
\end{aligned}
$$

- To avoid confusion the asterisks have been deleted although only the labeled species is under consideration here.
Substituting the value for $\frac{\mathrm{dE}}{\mathrm{dt}}$ determined from the relation, $\frac{d P}{d t}+\frac{d A}{d t}+\frac{d E}{d t}=0$, and the value for $\frac{\mathrm{dP}}{\mathrm{dt}}$ determined from the derivative of $(1)$ and collecting terms,

$$
\begin{aligned}
\frac{d^{3} A}{d t^{3}}+ & \frac{d^{2} A}{d t^{2}}\left(\lambda_{a p}+\lambda_{p q}+\lambda_{e p}+\lambda_{p e}\right) \\
& +\frac{d A}{d t}\left(\lambda_{p a} \lambda_{e p}+\lambda_{a p} \lambda_{e p}+\lambda_{a p} \lambda_{p e}\right)=0
\end{aligned}
$$

To solve for A we find the value of $\lambda$ so that ( 6 ) will be satisfied by a solution of the form

$$
\mathrm{A}=\mathrm{ce}^{\lambda t}
$$

Differentiating (7) and substituting in (6) reduces (6) to a cubic equation in $\lambda$ which has three roots,

$$
\begin{aligned}
\lambda_{1}, \lambda_{2}=\frac{1}{2}\{ & -\left(\lambda_{\mathrm{ap}}+\lambda_{\mathrm{pa}}+\lambda_{\mathrm{ep}}+\lambda_{\mathrm{pe}}\right) \\
& \pm\left[\left(\lambda_{\mathrm{ap}}+\lambda_{\mathrm{pa}}+\lambda_{\mathrm{ep}}+\lambda_{\mathrm{pe}}\right)^{2}\right. \\
& \left.\left.-4\left(\lambda_{\mathrm{pa}} \lambda_{\mathrm{ep}}+\lambda_{\mathrm{ap}} \lambda_{\mathrm{ep}}+\lambda_{\mathrm{ap}} \lambda_{\mathrm{pe}}\right)\right]^{\sharp}\right\} \\
\lambda_{3}=0 &
\end{aligned}
$$

The general solution for $A$ is, then,

$$
A=c_{1} e^{-\lambda_{1} t}+c_{2} e^{-\lambda_{2} t}+c_{8}
$$

which contains three essential arbitrary constants and in which the values for $\lambda$ are the negatives of those given above. Similarly

$$
\begin{aligned}
& P=m_{1} e^{-\lambda_{1} t}+m_{2} e^{-\lambda_{2} t}+m_{3} \\
& E=k_{1} e^{-\lambda_{1} t}+k_{2} e^{-\lambda_{2} t}+k_{3}
\end{aligned}
$$

At $t_{\infty}, A, P$, and $E$ take on their $\infty$ values, Therefore $c_{3}=A_{\infty}, m_{3}=P_{\infty}, k_{3}=E_{\infty}$. Since $A_{0}=A+P+E=A_{\infty}+P_{\infty}+E_{\infty}$, the sum of equations $(9),(10),(11)$ gives

$$
\begin{aligned}
& \left(c_{1}+m_{1}+k_{1}\right) e^{-\lambda_{1} t} \\
& +\left(c_{2}+m_{2}+k_{2}\right) e^{-\lambda_{2} t}=0,
\end{aligned}
$$

which is satisfied for all values of $t$ only by

$$
\begin{aligned}
& c_{1}+m_{1}+k_{1}=0 \\
& c_{2}+m_{2}+k_{2}=0
\end{aligned}
$$

Equations (9), (10), (11) may then be rewritten in the form given in the body of the paper,

$$
\begin{aligned}
& A=A_{\infty}+c_{1} e^{-\lambda_{1} t}+c_{2} e^{-\lambda_{2} t} \\
& P=P_{\infty}-\left(k_{1}+c_{1}\right) e^{-\lambda_{1} t} \\
& E=E_{\infty}+k_{1} e^{-\lambda_{1} t}+k_{2} e^{-\lambda_{2} t}
\end{aligned}
$$


From (1) and (13)

$\frac{\mathrm{dA}}{\mathrm{dt}}=-\lambda_{\mathrm{ap}} \mathrm{A}+\lambda_{\mathrm{pa}} \mathrm{P}=-\lambda_{1} \mathrm{c}_{1} \mathrm{e}^{-\lambda_{1} \mathrm{t}}$

$-\lambda_{2} c_{2} e^{-\lambda_{2} t}$

at $\mathrm{t}=\mathbf{0}$

$$
\frac{\mathrm{dA}}{\mathrm{dt}}=-\lambda_{\mathrm{ap}} \mathrm{A}_{0}=-\lambda_{1} \mathrm{c}_{1}-\lambda_{2} \mathrm{c}_{2}
$$

Similarly from (2) and (14) at $\mathrm{t}=0$

$$
\frac{\mathrm{dP}}{\mathrm{dt}}=\lambda_{\mathrm{sp}} \mathrm{A}_{0}=\lambda_{1}\left(\mathrm{k}_{1}+\mathrm{c}_{1}\right)+\lambda_{2}\left(\mathrm{k}_{2}+\mathrm{c}_{2}\right)
$$

Then from (17) and (18),

$$
\mathrm{k}_{1}=-\frac{\lambda_{2}}{\lambda_{1}} \mathrm{k}_{2}
$$

From (15), at $\mathrm{t}=0$

$$
\mathrm{E}_{0}=0=\mathrm{E}_{\infty}+\mathrm{k}_{1}+\mathrm{k}_{2}
$$

Solving for $k_{1}$ and $k_{2}$ from (19) and (20)

$$
\begin{aligned}
\mathrm{k}_{1} & =-\frac{\lambda_{2} \mathrm{E}_{\infty}}{\lambda_{2}-\lambda_{1}} \\
\mathrm{k}_{2} & =\frac{\lambda_{1} \mathrm{E}_{\infty}}{\lambda_{2}-\lambda_{1}}
\end{aligned}
$$

From (13), at $\mathrm{t}=0$

$$
A_{0}=A_{\infty}+c_{1}+c_{2}
$$

From (17) and (23),

$$
\begin{aligned}
& c_{1}=\frac{\lambda_{2}\left(A_{0}-A_{\infty}\right)-\lambda_{\mathrm{ap}} A_{0}}{\lambda_{2}-\lambda_{1}} \\
& c_{2}=-\frac{\lambda_{1}\left(A_{0}-A_{\infty}\right)-\lambda_{\mathrm{ap}} A_{0}}{\lambda_{2}-\lambda_{1}}
\end{aligned}
$$

\section{APPENDIX $B^{5}$}

Evaluation of the rate constants of iodoalbumin transfer from the values obtained in the graphical analysis

It is desired to express each rate constant in terms of the coefficients and exponents of equations (13), (14), and (15) in Appendix A.

Substituting in (1), the values for $A, P$, and $\frac{\mathrm{dA}}{\mathrm{dt}}$ derived from (13) and (14) of Appendix A,

$$
\begin{gathered}
-c_{1} \lambda_{1} \mathrm{e}^{-\lambda_{1} t}-c_{2} \lambda_{2} \mathrm{e}^{-\lambda_{2} t}=-\lambda_{2 p}\left(\mathrm{~A}_{\infty}^{*}\right. \\
\left.+\mathrm{c}_{1} \mathrm{e}^{-\lambda_{1} t}+\mathrm{c}_{2} \mathrm{e}^{-\lambda_{2} t}\right)+\lambda_{\mathrm{pa}}\left\{\mathrm{P}_{\infty}^{*}\right. \\
\left.-\left(\mathrm{c}_{1}+\mathrm{k}_{1}\right) \mathrm{e}^{-\lambda_{1} \mathrm{t}}-\left(\mathrm{k}_{2}+\mathrm{c}_{2}\right) \mathrm{e}^{-\lambda_{2} t}\right\}
\end{gathered}
$$

- The asterisks have been reintroduced here to differentiate labeled from unlabeled albumin.
Collecting terms,

$$
\begin{aligned}
& \mathrm{e}^{-\lambda_{1} t}\left\{-c_{1} \lambda_{1}+c_{1} \lambda_{2 p}+\left(c_{1}+k_{1}\right) \lambda_{p 2}\right\} \\
&=e^{-\lambda_{2} t}\left\{c_{2} \lambda_{2}-c_{2} \lambda_{a p}-\left(k_{2}+c_{2}\right) \lambda_{p a}\right\} \\
&-\lambda_{a p} A_{\infty}^{*}+\lambda_{p a} P_{\infty}^{*}
\end{aligned}
$$

Since, from the equilibrium conditions,

$$
-\lambda_{\mathrm{ap}} \mathrm{A}_{\infty}+\lambda_{\mathrm{pa}} \mathrm{P}_{\infty}^{*}=0,
$$

equation (2) is satisfied for all values of $t$ only if

$$
\begin{aligned}
& c_{1} \lambda_{\mathrm{ap}}+\left(c_{1}+k_{1}\right) \lambda_{\mathrm{pa}}-c_{1} \lambda_{1}=0 \\
& c_{2} \lambda_{\mathrm{ap}}+\left(c_{2}+k_{2}\right) \lambda_{\mathrm{pa}}-c_{2} \lambda_{2}=0
\end{aligned}
$$

Solving for $\lambda_{\mathrm{pa}}$ and $\lambda_{\mathrm{ap}}$,

$$
\begin{aligned}
& \lambda_{\mathrm{ps}}=\frac{c_{1} c_{2}\left(\lambda_{1}-\lambda_{2}\right)}{c_{2} k_{1}-c_{1} k_{2}} \\
& \lambda_{\mathrm{ap}}=\lambda_{1}-\frac{\left(c_{1}+k_{1}\right) c_{2}\left(\lambda_{1}-\lambda_{2}\right)}{c_{2} k_{1}-c_{1} k_{2}}
\end{aligned}
$$

Similarly,

$$
\begin{aligned}
& \lambda_{\mathrm{pe}}=\frac{\mathrm{k}_{1} \mathrm{k}_{2}\left(\lambda_{1}-\lambda_{2}\right)}{c_{1} \mathrm{k}_{2}-\mathrm{c}_{2} \mathrm{k}_{1}} \\
& \lambda_{\mathrm{ep}}=\lambda_{1}-\frac{\left(\mathrm{k}_{1}+\mathrm{c}_{1}\right) \mathrm{k}_{2}\left(\lambda_{1}-\lambda_{2}\right)}{\mathrm{c}_{1} \mathrm{k}_{2}-\mathrm{c}_{2} \mathrm{k}_{1}}
\end{aligned}
$$

The values obtained by graphical analysis (Figure 3),

$$
\lambda_{1}=.133 \mathrm{day}^{-1} ; \lambda_{2}=.562 \mathrm{day}^{-1} ;
$$

$$
\mathrm{c}_{1}=.465 ; \mathrm{c}_{2}=.060 ; \mathrm{k}_{1}=-.390, \mathrm{k}_{2}=.095
$$

are substituted in (5), (6), (7), and (8). Then

$$
\begin{aligned}
& \lambda_{\mathrm{ap}}=.102 \mathrm{day}^{-1} \\
& \lambda_{\mathrm{pa}}=.178 \mathrm{day}^{-1} \\
& \lambda_{\mathrm{po}}=.236 \mathrm{day}^{-1} \\
& \lambda_{\mathrm{op}}=.178 \mathrm{day}^{-1}
\end{aligned}
$$

When these values for the transfer rate constants are substituted in equation ( 8 ) of Appendix $A$, the values obtained for $\lambda_{1}$ and $\lambda_{2}$,

$$
\begin{aligned}
& \lambda_{1}=.130 \text { day }^{-1} \\
& \lambda_{2}=.564 \text { day }^{-1}
\end{aligned}
$$

agree with the graphical values used.

Employing the values for $\mathrm{A}, \mathrm{P}$, and $\mathrm{E}$ at the beginning of the experiment, albumin transfer from ascitic fluid to plasma is, then,

$$
\lambda_{\mathrm{ap}} \mathrm{A}=\left(.102 \mathrm{day}^{-1}\right)(218 \mathrm{Gm} .)=22 \mathrm{Gm} . / \text { day }
$$

Similarly,

Albumin transfer from plasma to ascitic fluid $=(.178)(137)=24 \mathrm{Gm} . /$ day 
Albumin transfer E.V. space to

$$
\text { plasma }=(.178)(157)=28 \mathrm{Gm} . / \text { day }
$$

Albumin transfer plasma to E.V.

$$
\text { space }=(.236)(137)=32 \mathrm{Gm} . / \text { day }
$$

Discrepancies arising from the absence of strict steady state conditions are discussed in the body of the paper.

\section{ACKNOWLEDGMENTS}

We are indebted to Dr. Lynn Fulkerson for obtaining the ascitic fluid samples and to Mr. Paul Newman for the illustrations. Thanks are also due to Mrs. Florence Cavanagh and Mrs. Frieda Steiner for secretarial assistance.

\section{REFERENCES}

1. Schoenberger, J. A., Kroll, G., Sakamoto, A., and Kark, R. M., Investigation of the permeability factor in ascites and edema using albumin tagged with $\mathrm{I}^{121}$. Gastroenterology, 1952, 22, 607.

2. Berson, S. A., Yalow, R. S., Schreiber, S. S., and Post, J., Tracer experiments with I'si labeled human serum albumin: Distribution and degradation studies. J. Clin. Invest., 1953, 32, 746.

3. Kingsley, G. R., A rapid method for the separation of serum albumin and globulin. J. Biol. Chem., 1940, 133, 731.

4. Berson, S. A., and Yalow, R. S., The use of $\mathrm{K}^{12}$ or $\mathrm{P}^{32}$ labeled erythrocytes and I's1 tagged human serum albumin in simultaneous blood volume determinations. J. Clin. Invest., 1952, 31, 572.

5. Sheppard, C. W., and Householder, A. S., The mathematical basis of the interpretation of tracer experiments in closed steady-state systems. J. Appl. Phys., 1951, 22, 510.

6. Schoenberger, J. A., Kark, R. M., Kroll, G., and Eckert, E., Endothelial permeability in man studied with albumin ${ }^{121}$ injected intravenously or intraperitoneally. J. Clin. Invest., 1953, 32, 601 (abst.)

7. Krogh, A., The Anatomy and Physiology of Capillaries, rev. and enl. ed., New Haven, Yale University Press, 1929.

8. Bauer, F. K., Blahd, W. H., and Fields, M., Ascitic fluid and plasma protein exchange. Studies with radioiodinated human serum albumin. J. Clin. Invest., 1953, 32, 553 (abst.). 